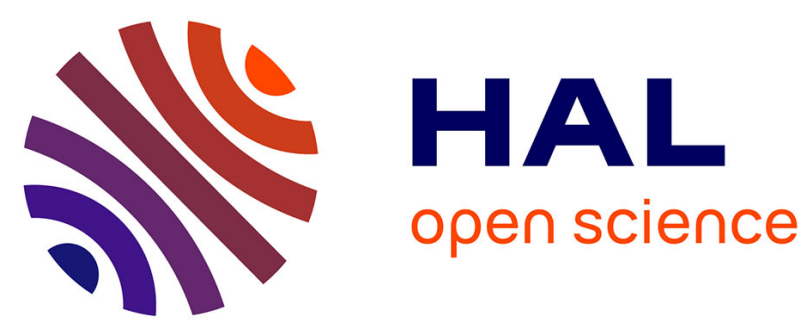

\title{
International Trade and Financial Integration : a Weighted Network Analysis
}

Giorgio Fagiolo, Javier Reyes, Stefano Schiavo

\section{To cite this version:}

Giorgio Fagiolo, Javier Reyes, Stefano Schiavo. International Trade and Financial Integration: a Weighted Network Analysis. 2007. hal-00973118

\section{HAL Id: hal-00973118 \\ https://hal-sciencespo.archives-ouvertes.fr/hal-00973118}

Preprint submitted on 3 Apr 2014

HAL is a multi-disciplinary open access archive for the deposit and dissemination of scientific research documents, whether they are published or not. The documents may come from teaching and research institutions in France or abroad, or from public or private research centers.
L'archive ouverte pluridisciplinaire HAL, est destinée au dépôt et à la diffusion de documents scientifiques de niveau recherche, publiés ou non, émanant des établissements d'enseignement et de recherche français ou étrangers, des laboratoires publics ou privés. 


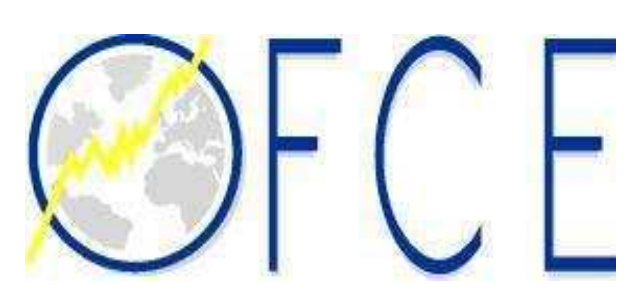

\title{
Document de travail
}

\section{International Trade and Financial Integration: a Weighted Network Analysis}

\author{
$\mathrm{N}^{\circ} 2007-11$
}

March 2007

Giorgio FAGIOLO

Sant'Anna School of Advanced Studies

Javier REYES

University of Arkansas

Stefano SCHIAVO

OFCE

Observatoire Français des Conjonctures Economiques

69, Quai d'Orsay 75340 Paris Codes 07

Tel : 0144185400 Fax:01 45560615

E-mait: ofce@oice sciences-poif Web htipl/wwwiofceisciences-poff: 


\title{
International Trade and Financial Integration: a Weighted Network Analysis
}

\author{
Giorgio Fagiolo* Javier Reyes ${ }^{\dagger} \quad$ Stefano Schiavo ${ }^{\ddagger}$
}

This version: February, 282007

\begin{abstract}
In this paper we compare the degree and patterns of trade and financial integration exploiting network analysis. We start from a simple binary analysis and then move to a more appropriate weighted approach, presenting a detailed overview of international goods and financial markets integration, and compare their main characteristics. Moving from binary to weighted analysis changes considerably the properties of the networks, and with them the picture of the integration process. Limiting to a binary approach can thus lead to a misrepresentation of the underlying economic phenomena. We find that the trade network is almost fully connected while international financial integration is less pervasive. Also, regional links continue to play a relevant role.
\end{abstract}

Keywords: International Trade, International Financial Flows, Globalization, Complex Weighted Networks, Dynamics.

JEL Classification: F10, F36, F40, G15.

*Laboratory of Economics and Management, Sant'Anna School of Advanced Studies, Pisa - Italy; e-mail: giorgio.fagiolo@sssup.it

${ }^{\dagger}$ Department of Economics, Sam M. Walton College of Business, University of Arkansas - U.S.A.; e-mail: JReyes@walton.uark.edu

$\ddagger$ Observatoire Français des Conjonctures Économiques, Département de recherche sur l’Innovation et la Concurrence, Valbonne - France; e-mail: stefano.schiavo@ofce.sciences-po.fr 


\section{Motivations and Goals}

The paper explores the patterns of international trade and financial integration adopting weighted network analysis as introduced in Barrat et al. (2004), Barrat et al. (2005) and Barthélemy et al. (2005).

A number of recent papers has studied the topological properties of the International Trade Network (ITN), but has only relied on binary (unweighted) analysis (Serrano \& Boguñá 2003, Garlaschelli \& Loffredo 2004, 2005). This means that a link between any two countries in the network is either in place or not depending on whether the trade flow is greater than some given threshold. Thus all links are treated the same way, i.e. they have the same impact on the properties of the network irrespective of the actual amount of trade that flows over them.

The first contribution of the paper is represented by the extension of the analysis to weighted networks, when each link is weighted by the amount of trade that it channels.

Second, we do not limit ourselves to the ITN, but for the first time study also bilateral (international) financial positions, comparing the properties of the International Financial Network (IFN) with those of the ITN in order to assess similarity and differences in goods and financial markets integration. In both cases we find that it is indeed important to use a weighted approach as the properties of the network change considerably with respect to the simple binary analysis.

The paper is organized as follows: section 2 reviews the relevant empirical literature on network analysis, section 3 briefly discusses the methodology and our research questions, while section 4 presents the data. Results are discussed in section 5 while section 6 concludes and offers some suggestions for future research. 


\section{A Glance at Existing Literature}

A recent wave of research has used network analysis to analyze a number of issues, from airplane traffic to research co-operation to the World Wide Web. Most of them belong to the physics domain and therefore focus mainly on the mechanics of the network, with little or no interest in the social phenomena (notably the economics) that underlie it.

On the other hand, a rather long tradition exists in sociology of using social network to investigate international trade relations. The common ground between sociology and network analysis is the idea that relational variables are more relevant (or at least as relevant as) individual characteristics to explain social phenomena. Most sociological papers have the goal of validating 'world-system' or 'dependency' theories, i.e. the notion that one can distinguish among 'core' and 'peripheral' countries, with the former appropriating most of the surplus value added produced in the latter, which in turn are thus prevented from developing.

In what follows we review the most relevant attempts in these two fields, plus a couple of recent economics papers that apply network analysis to study globalization and related phenomena and are therefore similar to our work in spirit.

\subsection{Papers in the sociology domain}

Snyder \& Kick (1979) start from the core/periphery setup postulated by dependency theories of differential economic growth. Trade is only one of the criteria used to classify a country into one of the three groups identified by the authors (core, periphery and semi-periphery). Results for international trade yield a clear-cut three-tiered structure with core countries nearly identified by OECD membership.

Breiger (1981) focuses on trade in 4 commodity classes, hence uses data on trade in particular products rather than aggregated data. Core countries (the exploiters) trade sophisticated industrial goods, while peripheral states trade commodities and row materials. The situation changes quite a bit when data are weighted: what emerges is the presence of two competing blocks, one dominated by the US (and comprising Canada 
and Japan) and the other represented by the then young (and much smaller) European Economic Community.

Smith \& White (1992) is the first paper to investigate the dynamics of the trade network by studying trade flow in three different time instances, 1965, 1970 and 1980. The analysis addresses the role of countries in the global division of labor, i.e. the level of export processing. While the authors report the existence of a continuum of countries from periphery to core, they still identify three main groups and some subblocks. Over time they document an enlargement of the core with the emergence of competitive centers. Also, within core distance is reduced and there are more upward than downward movements in the ranking. The main difference between core and non-core countries is the amount of intra-group trade, which is very large for the former, minuscule for the latter. Also, capital intensive goods dominate intra-core trade, while food exports characterize peripheral countries. A second, parallel phenomenon is the progressive marginalization of very peripheral countries.

The main research questions that drive the paper by Kim \& Shin (2002) make it our work: study the nature and the effects of globalization and the concurrent phenomenon of regionalization. Focusing on asymmetric links Kim \& Shin (2002) wish to discover the existence of a core/periphery setup: with core countries more likely to initiate the link, while peripheral ones to receive it. Centralization has decreased over time, so that globalization seems not to be associated with the emerging of a dominant center, but at the same time the paper highlights that the variance of degrees goes up as globalization is not even and therefore generates more heterogeneity.

\subsection{Papers in the physics domain}

Contributions in this area often refer to complex networks, i.e. network showing the following properties: (i) scale-free degree distribution with $P(k) \sim k^{-\gamma}$ and $2<\gamma \leq 3$; (ii) small world property, i.e. the fact that the average path length between any pair of vertices grows logarithmically with the size of the network coupled with a high clustering coefficient; (iii) degree-degree correlation (that can be assortative or disassortative 
depending on the tendency of highly connected nodes to attach to highly Vs. poorly connected ones). Within these works a few have used the world trade network or web (WTW) as a relevant example.

Serrano \& Boguñá (2003) study import and export flows for 179 counties in 2000 using a binary directed network (BDN) approach. Empirical analysis confirms the existence of a scale-free degree distribution implying high heterogeneity among actors. There is also a large (0.65) correlation between the number of trade links and per capita GDP. The clustering coefficient depends strongly on the vertex's degree, thus suggesting a hierarchy in the network, a finding that is confirmed by the fact that the average nearest network degree (ANND) depends also on the vertex's degree. In particular, the fact that the relation is decreasing suggests that highly interconnected countries tend to connect to poorly related ones in a hierarchical way.

Garlaschelli \& Loffredo (2004) aims at providing evidence in favor of so called hidden variable models whereby the topological properties of the network are determined by a single (fitness) variable, i.e. the potential ability of being connected. Specifically the authors postulate that GDP plays this role in the case of the WTW. With respect to the distribution of the degree $k(x)$ Garlaschelli \& Loffredo (2004) claim that it is not a power law and therefore the WTW is not a scale-free network. On the other hand, they agree with Serrano \& Boguñá (2003) in finding both disassortativity and that the clustering coefficient is decreasing in $k$, suggesting the presence of a hierarchical structure.

Serrano et al. (2006) build a weighted directed network of trade imbalances with links directed from net importers to net exporters. The probability distribution of imbalances is heavy-tailed and suggests the presence of large heterogeneity: only a small part of relations carry most of trade imbalances. ${ }^{1}$

\footnotetext{
${ }^{1}$ This feature may heavily depend on the use of net versus gross flows: in fact, since running a trade deficit implies receiving credit from partners makes it more likely that only large countries, wealthy countries, or countries whose currency is largely accepted as an international means of payment can afford to do so.
} 


\subsection{Papers in the economics domain}

Kastelle et al. (2006) represents a recent attempt to analyze globalization from an economic point of view using network analysis. The authors find that the density of the network is stable over time, but recognize that this is not very meaningful as the umber of countries has doubled. On the contrary, the average degree has grown from 11 to 20 . A possible limitation is the difficulty to study the interconnectedness of a graph in a meaningful way using binary analysis as all links are treated in the same way. They also claim that the remarkable stability of the clustering coefficient testifies against complete globalization, though their distinction between globalization and regionalization risks to be an artificial juxtaposition as the two phenomena needs not to be mutually exclusive; moreover unweighted analysis seems ill-suited for their purpose.

A second paper aiming at analyzing the process of economic globalization using a network approach is Kali \& Reyes (2007). Interestingly, although the starting point is very different from the papers in the sociology domain reviewed above, they also conclude that global trade is hierarchical with a core-periphery structure. Moreover, globalization and regionalization seem to coexist as integration of smaller countries into the network has increased considerably during the last decade of the XX century, but still trade patterns are strongly determined by geographical proximity, as usually found in gravity models. Kali \& Reyes (2007) find also that a country's position in the network has substantial implications for economic growth and has also a good potential for explaining episodes of financial contagion.

\section{$3 \quad$ Research Questions and Methodology}

In this section we identify the set of research questions representing the thread of the paper and guiding our analysis of both the ITN and the IFN. We will perform a comparative analysis aiming at shedding some lights on similarity and differences between 
goods and financial markets integration.

Also, throughout the paper we consistently compare results from binary and weighted network analysis: in this way we can compare our results to those existing in the literature, but above all show how results change once we weight flows by their intensity.

The last methodological point concerns the choice between directed and undirected analysis. Weighted network analysis has been done so far in the framework of undirected weighted graphs, i.e. graphs where the weights were symmetric with respect to nodes (see e.g. Barrat et al. 2005, Barthélemy et al. 2005). In other words, if $i$ and $j$ are any two nodes, and the weights attached to edges were labeled by $\omega_{. .}$, then $\omega_{i j}=\omega_{j i}$. This means assuming that the importance of the flow does not depend on the direction of the flow itself. Whether this assumption is justifiable or not is of course an empirical issue.

We have tested for symmetry in two ways: measuring the number of bilateral links in the network, and using a new measure based on the difference between the adjacency matrix and its transpose. Results (not reported but available upon request) suggest that the networks are sufficiently symmetric not to require a directed analysis. This is easily verified in the case of the binary trade matrix where more than $96 \%$ of links are bilateral. Rather high percentages are found also in the case of financial data, with around $75 \%$ of connections being reciprocated. ${ }^{2}$. In the case of weighted networks the asymmetry index is rather low and stable over the period under consideration, confirming that directed analysis is not called for. The values are again very small for trade flows, less so for what concerns financial data, which show also some heterogeneity across the different asset classes.

In sum, we in what follows we opt for an undirected approach whereby we do not distinguish flows going from A to B from those taking the opposite direction. Therefore we refer to binary and weighted undirected network analysis (BUN and WUN respectively), and leave directed analysis for further research.

The paper moves around four main research questions:

\footnotetext{
${ }^{2}$ This figure refers to the network generated by total portfolio asset holdings. The minimum proportion of bilateral links (just above 50\%) characterizes the IFN made by short-term debt (see section 4 for a detailed description of the data)
} 
Question 1 how much countries are connected among them? To answer we study the properties of the distributions of node degree and node strength, compute moments and look at how they change over time.

Question 2 are poor countries more/less connected? To assess this we compute the correlation between node degree (strength) and countries per capita GDP.

Question 3 do countries that are well connected have neighbors that are themselves well-connected (i.e. have many partners)? We address this question by means of average nearest-neighbor degree (ANND) or strength (ANNS) (see Garlaschelli \& Loffredo 2004, 2005). These coefficients measure for each node the average number (or weight) of neighbors of the neighbors of the node.

Question 4 are very connected countries (i.e. those which have many partners or high strength) linked with countries that are themselves tightly linked? This amounts to ask how much clustering is there and how it depends on node degree (strength).

\section{Data}

Our main constraint comes from financial data, which are taken from the IMF Coordinated International Portfolio Survey (CPIS). The survey was conducted for the first time in 1997 when it included only 29 economies participated; it has since then been replicated 4 times in the years 2001-2004 to include 71 countries and five data groups: total assets, equities, and debt, which is further separated into long- and short-term debt. It is to the data for 2001-2004 that we turn our attention in the analysis. Although this dataset does not allow one to track the evolution of the IFN over a long time span, it nonetheless provides one with some interesting insight on the patterns of financial integration.

Trade data have been assembled from the UN Comtrade database with the target of building a comparable network covering the same period and roughly the same countries as the CPIS. A perfect matching was not possible since the CPIS surveys a number of 
very small economies that are important financial centers (e.g. Cayman Islands, Jersey or Guernsey) but for which no trade data are available.

\section{Results}

Both the trade network and the finance network of total asset holdings have rather high density. In particular the ITN is almost fully connected; this is to say that countries in the sample have trade relation with almost everybody else. The percentage of existing links over the maximum possible number of relations $(n \cdot(n-1))$ reaches $98 \%$ for the ITN and ranges between 61 and $70 \%$ for the IFN. In this latter case then the density of the network has increased between 2001 and 2004 .

Other asset classes display lower densities, in particular the network made up of shortterm debt contracts has a density of $25-30 \%$ only. Cross-holding of equities is also less pervasive (48-56\%), while long-term debt contracts are substantially more widespread. The density of all financial networks has increased, although slightly, during the period under scrutiny.

The degree of a node represents the number of partners for each country present in the network and is the starting point of any binary network analysis. The mean (and median) degree is very high and stable for the ITN, lower but increasing for the IFN (both for total assets and for each asset class). Figure 1 and 2 display the distribution of node degree for the ITN and the IFN by means of a kernel density plot. The shape of the distributions does not change much over time, but is indeed very different for the two networks.

In the case of trade the vast majority of countries have a very large number of partners (due to data availability the maximum number of countries in the ITN is 64 in 2001 and 61 in 2004, which coincides with the mode of the distributions). Consistently with the fact that the network is almost full connected the degree distribution has most of the mass on the right tail, as nearly all countries trade with everybody else. For what concern the IFN, figure 2 shows a rather different picture. There in fact we see that the distribution 
Figure 1: Degree, kernel density plot: trade

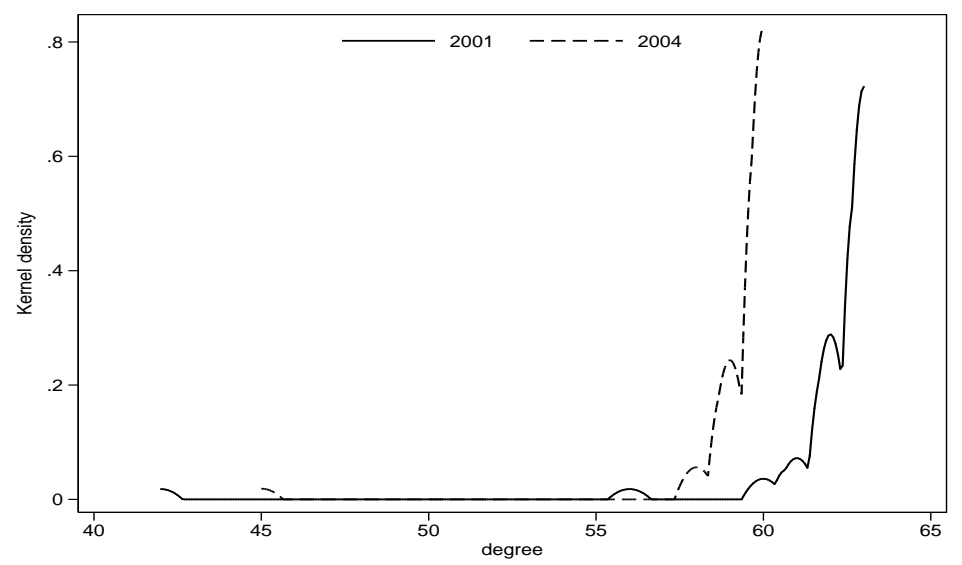

of node degree displays some bimodality with a first peak around 40 and a second modal value at the right tail of the distribution. Such a behavior, which is more pronounced in 2004 than at the beginning of the period, suggests the existence of three groups of countries: an elite of countries connected with everybody else, a larger group of countries with average connectedness and a periphery of less connected economies.

Figure 2: Degree, kernel density plot: total assets

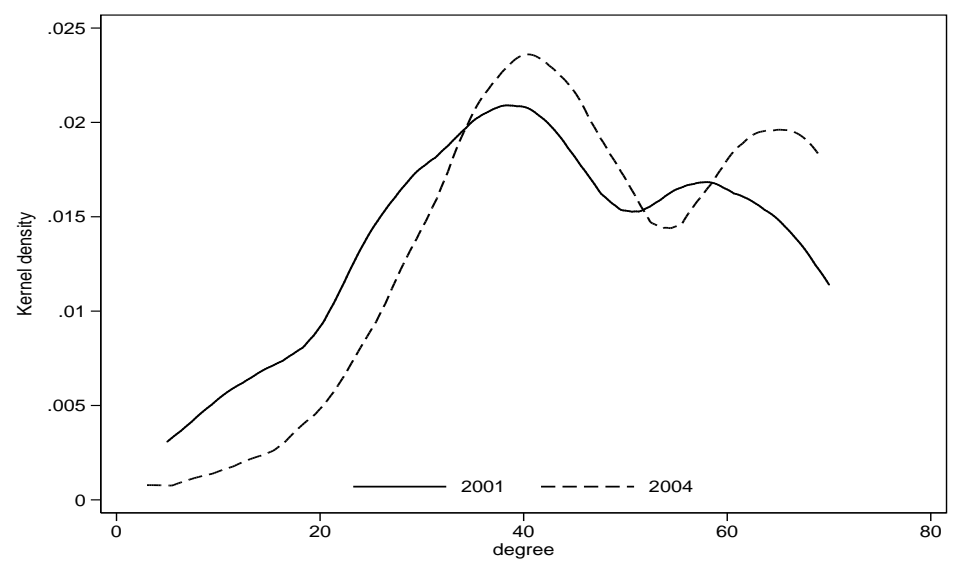

Over time it seems that more peripheral countries have made it to the middle group: in 2004 the distribution is more concentrated around the central peak and the left-hand tail leaner. Moreover, the coefficient of variation of node degree is smaller testifying for a lower dispersion. 
The picture changes significantly when we move from BUN to WUN analysis and weight each link by its intensity. This is to say that instead of analyzing a binary matrix made of zeros and ones we investigate the feature of a matrix whose elements are trade flows or asset holdings themselves. The concept of reference for WUN is node strength, which measures the intensity of the relationships of each country/node.

Figure 3: Strength, kernel density plot: trade

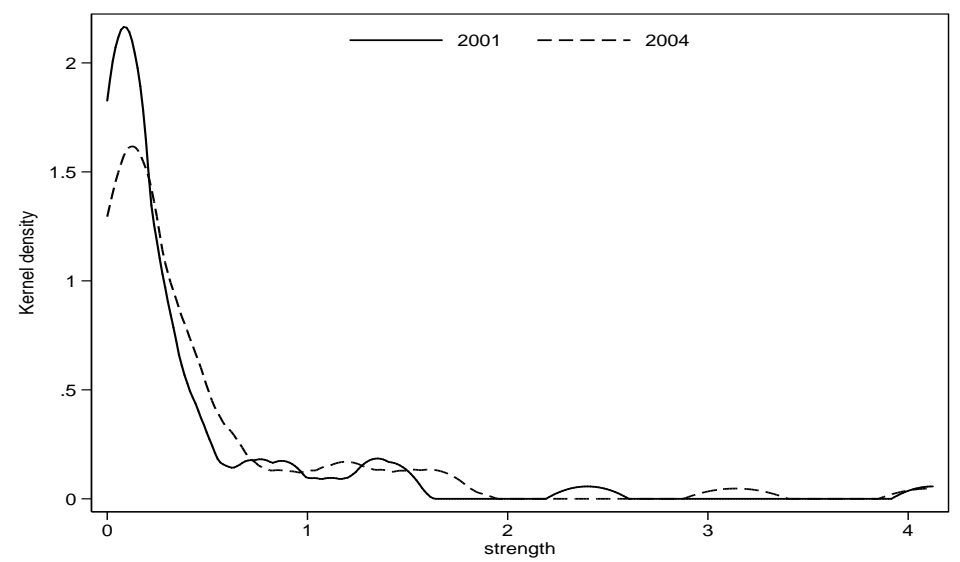

Figure 3 shows that the distribution of node strength for the ITN is no longer skewed on the left but rather on the right: most trade links carry very little weight, meaning that most commercial relations are feeble. This feature gets slightly reduced over time: in 2004 the peak is lower and more mass is distributed at higher values of strength. ${ }^{3}$

Something similar occurs in the case of the IFN (figure 4). As one could possibly guess, the distribution is even more heavily skewed here than in the case of trade. Any bimodality disappears: countries that hold assets of many partners do not have strong relationships with everyone.

Hence, the distributions of trade and financial linkages is completely different from those one would infer from simply observing the presence or absence of a relationship. This is an important methodological point as the vast majority of existing studies in network analysis relies on BUN.

Connectedness as measured by node degree and strength has increased for all asset

\footnotetext{
${ }^{3}$ The fact that the number of available countries in the network is smaller in 2004 surely plays a role in determining the reduction in the peak of the distribution.
} 
Figure 4: Strength, kernel density plot: total assets

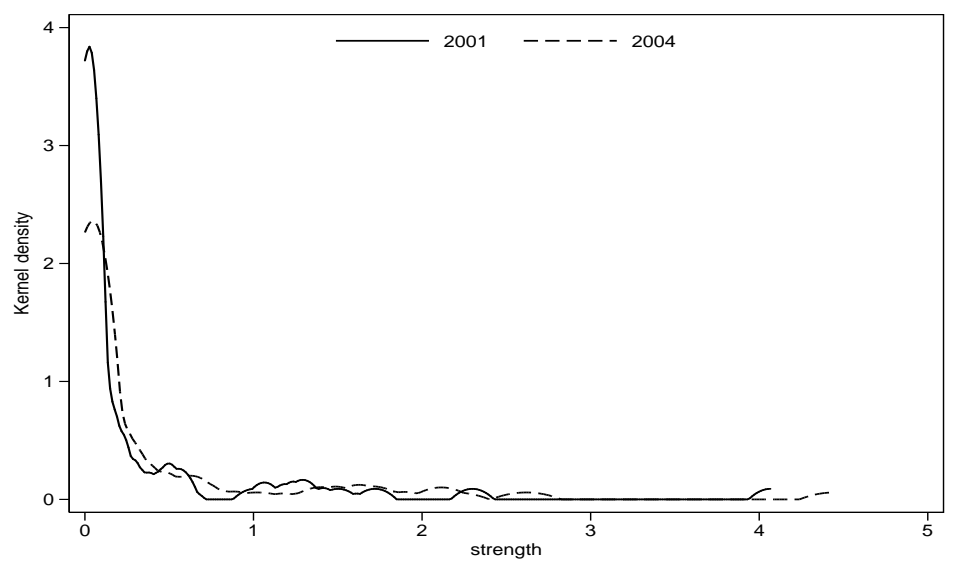

types. Apart for total assets, it is highest for debt contracts (total debt and long-term one) and lowest for short-term debt, with cross-border equity holdings somehow in between.

In the case of WUN, a second important piece of information is represented by disparity, which is nothing more than an Herfindahl index computed on node strength and therefore tells us whether trade and financial links carry similar weight or rather countries tend to display few tight links and a large number of feeble ones.

Table 1 reports the mean values of node disparity for the different types of WUN under scrutiny. One can see that in the case of ITN disparity is low and stable over time, but at the end of the sample period the IFN based on total assets has reached the same value though it started from a higher figure. The behavior of disparity for different asset classes is consistent with what we have found so far, with a higher value for equities, small figures for total and long-term debt and high disparity for short-term debt, again to testify a rather sparse network populated by links carrying very different weights.

Table 1: Node disparity

\begin{tabular}{lrrrr} 
& 2001 & 2002 & 2003 & 2004 \\
\hline trade & 0.130 & 0.125 & 0.122 & 0.117 \\
total assets & 0.207 & 0.215 & 0.159 & 0.117 \\
equities & 0.286 & 0.316 & 0.265 & 0.249 \\
debt & 0.203 & 0.220 & 0.200 & 0.172 \\
long-term debt & 0.200 & 0.215 & 0.198 & 0.182 \\
short-term debt & 0.356 & 0.393 & 0.334 & 0.294 \\
\hline
\end{tabular}


We turn now to analyze the different behavior of countries to see whether poor/rich countries are more or less connected. Table 2 helps us to see that once again BUN and WUN yield very different results and therefore weighting is indeed important to correctly investigate the behavior of the ITN and the IFN. The correlation coefficient between node degree and per capita GDP is low and never statistically significant, not even for the ITN. Hence we would conclude that there is not any relation between the position within the network (connectedness) and per capita GDP. The lower panel of table 2 displays the correlation coefficients for WUN. Contrary to the previous findings there is a positive and significant (at 1\%) correlation between node strength and per capita GDP. This is to say that once we control for the intensity of trade and financial connections we observe that richer countries are better connected than poorer economies. The correlation is lower in the case of trade than for finance and rather stable over time while different asset types do not display marked differences.

Table 2: Correlation between node degree/strength and per capita GDP

\begin{tabular}{ccccccc} 
& \multicolumn{3}{c}{ total } & long & short \\
debt & debsets & equities & debt & debt & BUN: degree - pcGDP \\
2001 & 0.156 & 0.015 & -0.043 & 0.037 & 0.031 & -0.045 \\
2002 & 0.189 & -0.027 & -0.076 & 0.008 & 0.013 & -0.017 \\
2003 & 0.197 & 0.013 & -0.072 & 0.045 & 0.035 & -0.025 \\
2004 & 0.182 & 0.000 & -0.093 & 0.073 & 0.076 & -0.026 \\
& \multicolumn{7}{c}{ WUN: strength - pcGDP } \\
2001 & 0.466 & 0.577 & 0.551 & 0.582 & 0.584 & 0.505 \\
2002 & 0.452 & 0.579 & 0.553 & 0.573 & 0.570 & 0.509 \\
2003 & 0.431 & 0.569 & 0.530 & 0.568 & 0.569 & 0.484 \\
2004 & 0.411 & 0.576 & 0.534 & 0.578 & 0.573 & 0.506 \\
\hline
\end{tabular}

The average nearest-neighbor degree (ANND) (see Garlaschelli \& Loffredo 2004, 2005, DeMontis et al. 2005) and the average nearest-neighbor strength (ANNS) allows one to assess the degree of assortativity that exists within the network. In other words, whether countries choose as partners nations with the same degree (strength) and whether the relationships with high/low degree countries (strength) follow the magnitude of the interaction.

Table 3 displays the correlation between node degree (strength) and ANND (ANNS). 
This time BUN and WUN analysis gives the same picture pointing toward a disassortative network; the only relevant change brought about by weighting concerns the size of the correlations which are reduced in the case of WUN.

Serrano \& Boguñá (2003) report a similar finding (disassortativity) for the ITN, a result that we can now extend to the IFN irrespective of the chosen asset type. Correlation coefficients are significantly different from zero both in the case of BUN and WUN. While in the binary case the IFN displays a very large value that is higher (in absolute value) than the corresponding figure for trade (apart from the usual case of short-term debt), weighting reverses the ranking and makes the ITN more disassortative than the IFN. This feature implies not only that poorly connected countries preferentially connect to high degree countries (BUN case), sometimes referred to as hubs, but also that the magnitudes of the interactions follow the same pattern (WUN).

Table 3: Correlation between node degree (strength) and ANND (ANNS)

\begin{tabular}{ccccccc}
\multicolumn{1}{c}{ total } & tong & $\begin{array}{c}\text { lonort } \\
\text { debt } \\
\text { debt }\end{array}$ \\
\hline \multicolumn{7}{c}{ BUN: degree - ANND } \\
2001 & -0.705 & -0.964 & -0.916 & -0.946 & -0.948 & -0.552 \\
2002 & -0.661 & -0.957 & -0.931 & -0.979 & -0.976 & -0.724 \\
2003 & -0.572 & -0.957 & -0.878 & -0.948 & -0.972 & -0.759 \\
2004 & -0.659 & -0.977 & -0.922 & -0.967 & -0.964 & -0.830 \\
\multicolumn{7}{c}{ WUN: strength - ANNS } \\
2001 & -0.607 & -0.423 & -0.401 & -0.495 & -0.515 & -0.323 \\
2002 & -0.513 & -0.392 & -0.418 & -0.463 & -0.488 & -0.277 \\
2003 & -0.494 & -0.531 & -0.459 & -0.596 & -0.577 & -0.373 \\
2004 & -0.679 & -0.556 & -0.485 & -0.581 & -0.576 & -0.376 \\
\hline
\end{tabular}

The behavior of financial links, in particular the strong negative correlation for BUN can be explained by the existence of a number of benchmark securities that enter almost every portfolio. When it comes to measuring the importance of such securities though, assets issued by more peripheral countries become appealing in view of diversification, so that weighted financial links become more dispersed and the relevance of hubs diminishes.

The last question on our list concerns clustering, which measures the local interconnectivity of the network by computing the number of complete triangles originating from a given country. In other words how many of the partners of country $i$ are themselves 
Table 4: Correlation between node degree (strength) and clustering

\begin{tabular}{ccccccc}
\multicolumn{8}{c}{ total } & long & $\begin{array}{c}\text { short } \\
\text { debt } \\
\text { debt }\end{array}$ \\
\hline \multicolumn{7}{c}{ BUN: degree - clustering } \\
2001 & -0.520 & -0.958 & -0.926 & -0.954 & -0.960 & -0.199 \\
2002 & -0.495 & -0.944 & -0.907 & -0.965 & -0.967 & -0.501 \\
2003 & -0.369 & -0.954 & -0.873 & -0.974 & -0.973 & -0.566 \\
2004 & -0.482 & -0.976 & -0.911 & -0.978 & -0.972 & -0.629 \\
\multicolumn{7}{c}{ WUN: strength - weighted clustering } \\
2001 & 0.985 & 0.973 & 0.958 & 0.965 & 0.969 & 0.549 \\
2002 & 0.984 & 0.963 & 0.945 & 0.972 & 0.983 & 0.690 \\
2003 & 0.976 & 0.977 & 0.949 & 0.981 & 0.986 & 0.775 \\
2004 & 0.973 & 0.979 & 0.952 & 0.987 & 0.983 & 0.781 \\
\hline
\end{tabular}

connected to each other.

The clustering coefficient (CC) that characterized BUN analysis is bounded between 0 and 1: it is almost 1 in the case of the ITN, consistently with the previous finding of a fully connected network, but values are quite high (in the .70-.85 range) for the IFN as well.

Table 4 reports the correlations between the CC and its weighted counterpart (WCC) and node degree and strength. Results for both the ITN and the IFN show that BUN and WUN analysis end up giving opposite results: while in fact the $\mathrm{CC}$ is negatively related to node degree, the WCC and node strength display a positive correlation. ${ }^{4}$ Hence, while BUN analysis suggests the presence of hierarchy in the networks, WUN rejects this hypothesis.

Hierarchy is a phenomenon whereby partners of well connected countries are less interconnected than partners of poorly connected ones. Out of the jargon, a perfect hierarchical network is one where all relations are unidirectional as in an organizational chart or a tree diagram. In economics hierarchy would signal the existence of a core-periphery structure similar to the one postulated by dependency theories we have encountered in section 2.1.

Table 4 shows that when we control for the intensity of trade and financial linkages not only does this hierarchical structure disappears, but we observe the opposite phenomenon. The correlation coefficient between node strength and the WCC turns positive and signif-

\footnotetext{
${ }^{4}$ In both cases the correlations are significant at $1 \%$.
} 
icant and testifies the fact that interconnected triples are more frequently composed by links with higher weights. This is sometimes referred to as a "rich club phenomenon"': clustering tend to be higher within groups of "strong"' nodes.

Figure 5 gives a pictorial description of the positive correlation between strength and weighted clustering in the case of trade and total asset holdings. We can see that the correlation is stronger in the case of trade in line with the higher connectedness of the ITN.

Figure 5: Scatterplot of WCC versus node strength, ITN and IFN
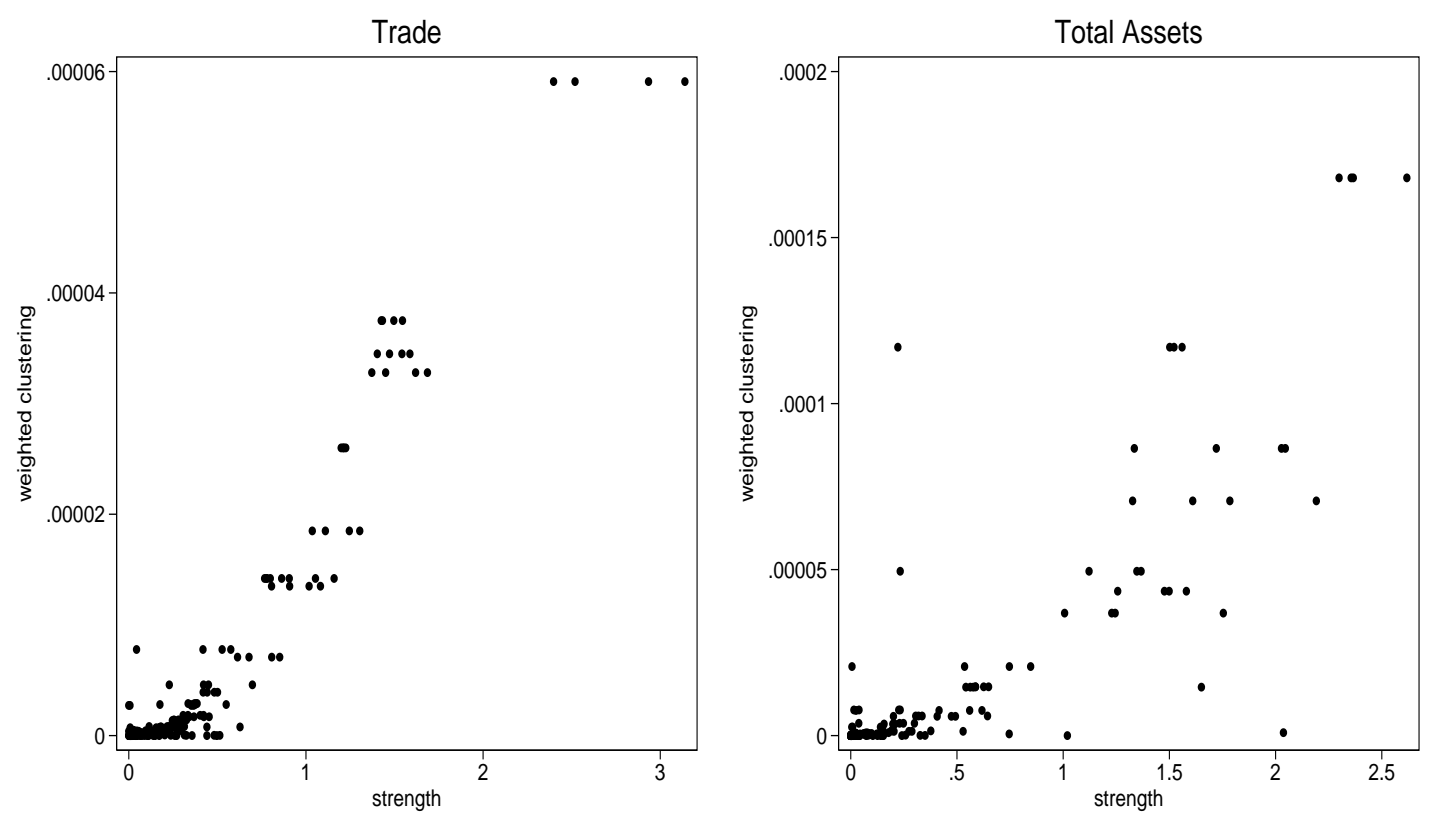

The behavior of different asset types is rather homogeneous and the size of the correlation coefficient does not vary a lot across different assets, with the exception of short-term debt, which displays values that are sensibly lower.

It is probably worth noting that the change in the sign of the correlations that we observed moving from BUN to WUN analysis is repeated when we relate the CC and WCC to per capita GDP. Hence, BUN tells that the partners of richer countries are less interconnected than those of poorer economies (the correlation is in fact negative). This feature suggests the existence of a hierarchical structure based this time not on the position within the network (i.e. the degree of connectedness), but rather on the degree 
of economic development (as measured by per capita income). On the contrary, weighted analysis states that the partners of countries characterized by a higher per capita GDP are more interconnected than those of poorer countries. A likely reason for this is the persistent relevance of regional integration (trade agreements being the utmost example): since these form of international co-operation usually take place among countries with similar degrees of development (think of the EU or Mercosur for instance), WUN analysis appears to capture the intensity of such preferential relations.

\section{Conclusions and Possible Extension}

The paper has investigated the properties of the ITN and the IFN using both a binary and a weighted network approach. From a methodological point of view we have shown the importance of weighting trade and financial relationships by their intensity. BUN analysis in fact can lead to a wrong description of the properties of the network, while the additional information used in WUN grants a cleaner and fuller understanding of the phenomena at stake. This represents a first important contribution of the paper.

Expectedly, the paper finds that the ITN is more densely connected than the IFN and that the degree of international financial integration varies with asset type: it is highest for long-term debt contracts, somewhat lower for equities and rather low for short-term debt, which is characterized by a sparse network.

Rich countries tend to be better linked and to form small groups of tightly connected economies. These cliques are formed along the lines of both connectedness and richness and can be seen as a sign of the persistent relevance of regionalization. However, the growing importance of global links is testified by the disassortative feature of both the ITN and the IFN: poorly connected nodes tend to connect to central ones and use them as hubs to access the rest of the network.

For what concerns future research, the next step calls for a directed network analysis in order to see whether our results are sensitive to this modification. 
Also, a natural extension of our work entails matching topological properties with country-specific variables to see how the structure of the ITN and IFN is shaped by (and shapes) macroeconomic variables such as growth. This goes in the direction of Kali \& Reyes (2007) who study how integration and financial crises can be explained by the topology of ITN.

Last, we also wish to explore the relation between the geographical net, i.e. the network generated by actual distances between countries and that implied by the trade and financial integrations in order to see whether access to world markets (globalization) is a substitute or a complement to regionalization. Along this line, an interesting question concerns the impact of regional integration (trade agreements, but also monetary integration) on the characteristics of the network.

\section{References}

Barrat, A., Barthélemy, M. \& Vespignani, A. (2005), Weighted evolving networks: coupling topology and weights dynamics, Technical Report 0401057v2, arXiv:cond-mat.

Barrat, A., Barthélemy, M., Pastor-Satorras, R. \& Vespignani, A. (2004), 'The architecture of complex weighted networks', Proceedings of the National Academy of Sciences 101, 3747-52.

Barthélemy, M., Barrat, A., Pastor-Satorras, R. \& Vespignani, A. (2005), 'Characterization and modeling of complex weighted networks', Physica A 346, 34-43.

Breiger, R. (1981), Structure of economic interdependence among nations, in P. M. Blau \& R. K. Merton, eds, 'Continuities in structural inquiry', Sage, Newbury Park, CA, pp. $353-80$.

DeMontis, A., Barthélemy, M., Chessa, A. \& Vespignani, A. (2005), The structure and evolution of inter-urban traffic: A weighted network analysis, Technical Report 0507106v2, arXiv:physics. 
Garlaschelli, D. \& Loffredo, M. (2004), 'Fitness-dependent topological properties of the world trade web', Physical Review Letters 93, 188701.

Garlaschelli, D. \& Loffredo, M. (2005), 'Structure and evolution of the world trade network', Physica A 355, 138-44.

Kali, R. \& Reyes, J. (2007), 'The architecture of globalization: A network approach to international economic integration', Journal of International Business Studies. Forthcoming.

Kastelle, T., Steen, J. \& Liesch, P. (2006), Measurig globalisation: an evolutionary economic approach to tracking the evolution of international trade, Paper presented at the DRUID Summer Conference on Knowledge, Innovation and Competitiveness: Dynamycs of Firms, Networks, Regions and Institutions - Copenhagen, Denemark, June $18-20$.

Kim, S. \& Shin, E.-H. (2002), 'A longitudinal analysis of globalization and regionalization in international trade: A social network approach', Social Forces 81, 445-71.

Serrano, A. \& Boguñá, M. (2003), 'Topology of the world trade web', Physical Review E 68, 015101(R).

Serrano, A., Boguñá, M. \& Vespignani, A. (2006), Commercial pathways and the global organization of the international trade system, Unpublished manuscript.

Smith, D. \& White, D. (1992), 'Structure and dynamics of the global economy: Network analysis of international trade, 1965-1980', Social Forces 70, 857-93.

Snyder, D. \& Kick, E. (1979), 'Structural position in the world system and economic growth 1955-70: A multiple network analysis of transnational interactions', American Journal of Sociology 84, 1096-126. 